\title{
Comparison of the International Association of the Diabetes and Pregnancy Study Group (IADPSG) criteria for diagnosis of gestational diabetes mellitus against a 'one-stop' diagnostic test: A Prospective Descriptive Cross Sectional study
}

\author{
Gardie Role Malwattage Udara Jayawardena ${ }^{1}$, Krishnapillai Guruparan ${ }^{1}$, Dulika \\ Sumathipala $^{2}$, Udagama Don Ratnasiri ${ }^{3}$, and Hemantha Senanayake ${ }^{4}$ \\ ${ }^{1}$ Postgraduate Institute of Medicine University of Colombo \\ ${ }^{2}$ University of Colombo Faculty of Medicine \\ ${ }^{3}$ Desoysa Hospital for Women, Sri Lanka \\ ${ }^{4}$ Affiliation not available
}

June 26, 2020

\begin{abstract}
Objective: The aim of this study was to investigate the utility of a single value non fasting glucose tolerance test as described by the Diabetes in Pregnancy Study Group of India (DIPSI) in detecting GDM Design: A Prospective Descriptive Cross Sectional study Setting: A Tertiary care maternity hospital in Sri Lanka. Sample: A sample of 165 pregnant women between 24-28 weeks of gestation was recruited Method: All women had the DIPSI and standard OGTT tests performed within a one week and results compared using standard non parametric tests. Main outcome measures:DIPSI and IADPSG criteria were compared using sensitivity, specificity and area under the ROC curve. Results: According to the IADPSG criteria $20 \%$ (33/165) had GDM, compared to $22.4 \%$ (37/165) detected by DIPSI. Sensitivity of DIPSI criteria was $64 \%$ while specificity was $88 \%$. The area under receiver operator curve was 0.8 . The mean satisfaction for DIPSI was of $8.9 \pm 0.4$, compared to 4.7 \pm 1.3 for OGTT $(\mathrm{p}<0.001)$. Analysis of false positives showed that it was mainly due to elevated fasting values in the IADPSG criteria. Conclusion: Although DIPSI has a low sensitivity compared to the IADPSG criteria, area under the ROC curve is 0.80 indicating its utility for diagnosing GDM. It has additional advantages of allowing a diagnosis of GDM in a single visit and high acceptability among women. Funding: This study was self-funded Key words : Gestational Diabetes Mellitus, Diabetes in Pregnancy Study Group India, Oral Glucose Tolerence Test
\end{abstract}

Comparison of the International Association of the Diabetes and Pregnancy Study Group (IADPSG) criteria for diagnosis of gestational diabetes mellitus against a 'one-stop' diagnostic test: A Prospective Descriptive Cross Sectional study

Jayawardena G.R.M.U.G.Pa,c

Guruparan $K^{\mathrm{a}, \mathrm{c}}$

Sumathipala W.L.D.S ${ }^{b}$

Ratnasisi U.D.P

Senanayake H.M

Post Graduate Institute of medicine, University of Colombo, Sri Lanka ${ }^{a}$ 
Faculty of Medicine, University of Colombo, Sri Lanka ${ }^{b}$

Desoysa Hospital for Women, Colombo, Sri Lanka ${ }^{c}$

Department of Obstetrics and Gynaecology, Faculty of Medicine, University of Colombo, Sri Lanka $^{\mathrm{d}}$

Corresponding author : GRMUGP Jayawardena

Ward 2, 5, 8

De Soysa Hospital for Women, Colombo 08, Sri Lanka

Phone: +94772987746

Email address: udarajayawardena@gmail.com

Running Title

Comparison of DIPSI test against IADPSG criteria

\section{Abstract}

\section{Objective:}

The aim of this study was to investigate the utility of a single value non fasting glucose tolerance test as described by the Diabetes in Pregnancy Study Group of India (DIPSI) in detecting GDM

Design: A Prospective Descriptive Cross Sectional study

Setting: A Tertiary care maternity hospital in Sri Lanka.

Sample: A sample of 165 pregnant women between 24-28 weeks of gestation was recruited

Method:

All women had the DIPSI and standard OGTT tests performed within a one week and results compared using standard non parametric tests.

\section{Main outcome measures:}

DIPSI and IADPSG criteria were compared using sensitivity, specificity and area under the ROC curve.

\section{Results:}

According to the IADPSG criteria 20\% (33/165) had GDM, compared to $22.4 \%(37 / 165)$ detected by DIPSI. Sensitivity of DIPSI criteria was $64 \%$ while specificity was $88 \%$. The area under receiver operator curve was 0.8 . The mean satisfaction for DIPSI was of $8.9 \pm 0.4$, compared to $4.7 \pm 1.3$ for OGTT $(\mathrm{p}<0.001)$. Analysis of false positives showed that it was mainly due to elevated fasting values in the IADPSG criteria.

\section{Conclusion:}

Although DIPSI has a low sensitivity compared to the IADPSG criteria, area under the ROC curve is 0.80 indicating its utility for diagnosing GDM. It has additional advantages of allowing a diagnosis of GDM in a single visit and high acceptability among women.

Funding: This study was self-funded

Key words: Gestational Diabetes Mellitus, Diabetes in Pregnancy Study Group India, Oral Glucose Tolerence Test

\section{Tweetable abstract}

Study investigates the utility of a single value non-fasting glucose tolerance test (DIPSI) in detecting GDM 


\section{Hosted file}

Main Text.docx available at https://authorea.com/users/337062/articles/462731-comparison-ofthe-international-association-of-the-diabetes-and-pregnancy-study-group-iadpsg-criteriafor-diagnosis-of-gestational-diabetes-mellitus-against-a-one-stop-diagnostic-test-aprospective-descriptive-cross-sectional-study

\section{Hosted file}

Tables.docx available at https://authorea.com/users/337062/articles/462731-comparison-ofthe-international-association-of-the-diabetes-and-pregnancy-study-group-iadpsg-criteriafor-diagnosis-of-gestational-diabetes-mellitus-against-a-one-stop-diagnostic-test-aprospective-descriptive-cross-sectional-study

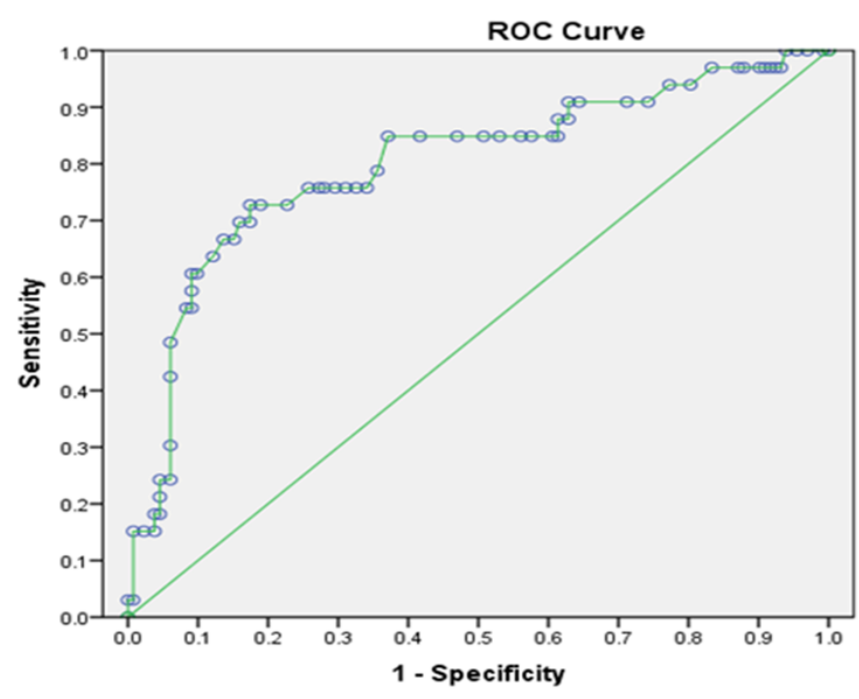

Source of the Curve

$\bigcirc$ DIPSI

Reference Line -

IADPSG 